\title{
COMMENT
}

\section{SAGE Ltd: Continuing the Journey to Transform Gender Equity, Diversity and Inclusion in Australia's Higher Education and Research Sector}

Wafa El-Adhami ${ }^{(\mathbb{D})}$ Chief Executive Officer of Science in Australia Gender Equity (SAGE) Limited, Australia

Keywords - SAGE, Athena Swan, gender equity, transparency, accountability

Acknowledgements: I am most thankful to the SAGE team for their contributions to the effective implementation of Athena Swan in Australia and their critical analysis of Institutional Bronze applications and to this piece. I acknowledge the commitment and immense body of work undertaken by SAGE participants on which the success of SAGE is founded. We thank the Australian Government for its grant funding support that secured SAGE financial viability and enabled its expansion.

Disclosure statement: The author has no conflicts of interests to report.

License: This work is under Attribution-NonCommercial-ShareAlike 4.0 International (CC BY-NC-SA 4.0) https://creativecommons.org/licenses/by-nc-sa/4.0/

Suggested citation: El-Adhami, W. (2021). "SAGE Ltd: Continuing the Journey to Transform Gender Equity, Diversity and Inclusion in Australia's Higher Education and Research Sector", Law in Context, 37 (2): 145-149, DOI: https://doi.org/10.26826/law$\underline{\text { in-context.v37i2.164 }}$

\section{Summary}

1. Introduction

2. Taking Action

3. The power of data and transparency

4. Demonstrating success

5. References

\section{INTRODUCTION}

Science in Australia Gender Equity (SAGE) began as a Pilot program in 2015. It was modelled on the UK's Athena Swan Charter (Advance HE), a framework for improving gender equity and diversity in science, technology, engineering, mathematics and medicine (STEMM); specifically, within higher education and research.

My first observation upon joining SAGE in 2016 was that the Athena Swan approach mirrors the proven quality, safety and performance accreditation frameworks in healthcare, and other comparable frameworks applied in manufacturing industries. The approach is fundamentally simple, but practically powerful in driving transformative change.

There are a number of key attributes that make these schemes effective:

- firstly, the systematic and iterative deployment 
of a scientific approach that relies on data, evidence and root cause analysis methodology to understand the "what and why" of issues and subsequently design the "how" to improve;

- secondly, the ongoing monitoring and measurement of outcomes and impact overseen by robust governance, transparency and accountability;

- thirdly, the establishment of industry/benchmarking standards for quality and performance that drive competition and therefore drive continuous improvement.

Combined with peer recognition and awards, these schemes contribute prestige and enhance reputationall of which are genuinely valued by universities and research institutions (Putting Gender on your Agenda 2018).

It is not surprising that Australia's higher education and research (HER) sector looked to the Athena Swan Charter. The sector is well accustomed to schemes like this, be it the regulatory type such as TEQSA's quality assurance for diversity, innovation and excellence, or voluntary national or international quality ranking schemes (for example, The Times Higher Education's World University Ranking). In fact, it is somewhat surprising that it took this long to see gender equity, diversity and inclusion in HER as integral to quality, safety, innovation and excellence. This is particularly so given the Athena Swan Charter principles (2021) revolve around creating equal access and participation in the workforce, supportive and safe workplaces, and inclusive organisational cultures, to encourage effective contributions from the full talent pool. This is eminently evident from a quick glance at the Charter principles, and the framework's systemic, structural and outcome-centred approach (Figure 1):

- Recognition and acknowledgement that an institution's systems, structures and culture pose serious barriers to gender equity, diversity and inclusion; as such, data (quantitative and qualitative) and evidence must drive a process of honest and reflective examination to identify solutions aimed at fixing these - not fixing women, minority genders and underrepresented groups;

- A commitment to action, systematically and at all levels, to drive change and transform an institution into an equitable, diverse, safe and inclusive workplace; action must be underpinned by active leadership, accountability and engagement; and

- Investment in systemic measurement and transparent reporting on performance, complemented by accountability measures to sustain improvement.

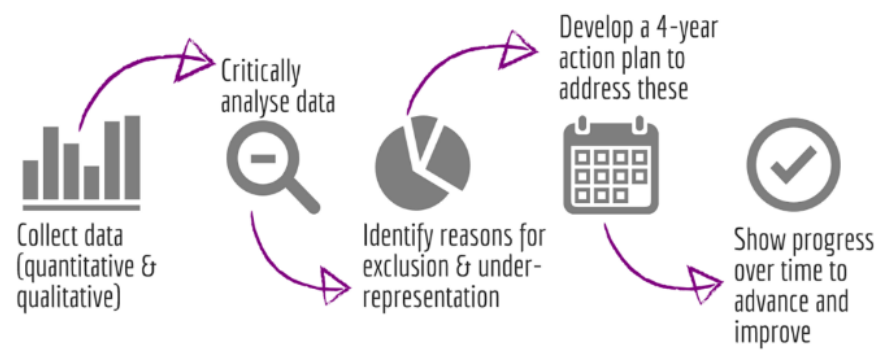

....... A scientific approach that is systematically driven

FIGURE 1: Athena Swan: a systematic process guided by scientific principles

\section{TAKING ACTION}

Leadership played a pivotal role in accelerating change in gender equity and diversity in STEMM nationally (Science in Australia Gender Equity Forum, Summary of Workshop Findings 2014). It galvanised commitment to establish the SAGE Pilot of Athena Swan as a partnership between the Australian Academy of Science (AAS) and the Australian Academy of Technology and Engineering (ATSE) (Putting Gender on your Agenda 2018). Such was the desire for action across the HER sector that 32 institutions signed up to the join the SAGE Pilot when launched in 2015-a commitment that involved financial and reputational risks for institutions, especially as accreditation applications and outcomes would be made public by SAGE. 
The sector's commitment attracted \$2 million in funding support from the Australian Government under the National Innovation and Science Agenda (FY 2016/17 to FY 2018/19). The momentum for change drew further interest from the sector, with SAGE participating institutions growing to 45 institutions within two years of the Pilot's launch. Overall, participation comprised $80 \%$ of Australia's universities; $50 \%$ of its publicly funded research agencies, and $12 \%$ of its medical research institutes.

\section{THE POWER OF DATA AND TRANSPARENCY}

In their respective applications for accreditation, SAGE institutions undertook extensive data collection and critical analysis to identify the institutional barriers that create and contribute to inequities.

Gender-disaggregated data were collected across the domains of system, structure and culture, in the areas of key career transition points, organisation and culture, career development and managing career breaks. Institutions examined these data organisation-wide and in individual sub-units or disciplines (focusing on STEMM). When considered alongside data on the institution's policies and practices, these data helped reveal, in rich detail, any barriers and issues at the institutional and/or local level. This in turn informed the design of targeted, context-specific actions.

Figure 2 provides an example from a SAGE analysis of institutional applications that helps explain the value of this approach. Overall, women are less likely to be recruited into STEMM positions than men are. In-depth analysis suggests that this is because women are less likely to apply for positions than men, with the proportion of women applicants decreasing as Level increases. When women do apply, however, they tend to be more successful than men. This suggests that, to improve the recruitment of women into STEMM positions, institutions should focus on increasing the number of women in the applicant pool, rather than designing interventions targeting the shortlisting or interview stages of the recruitment process.

Another important aspect of the approach lies in the active engagement with, and involvement of, those affected by institutional policies and practices. Qualitative data (Figure 3) are vital to understand the serious impact of the identified issues, support the case for change, and consequently mobilise collective action and accountability for organisational change.

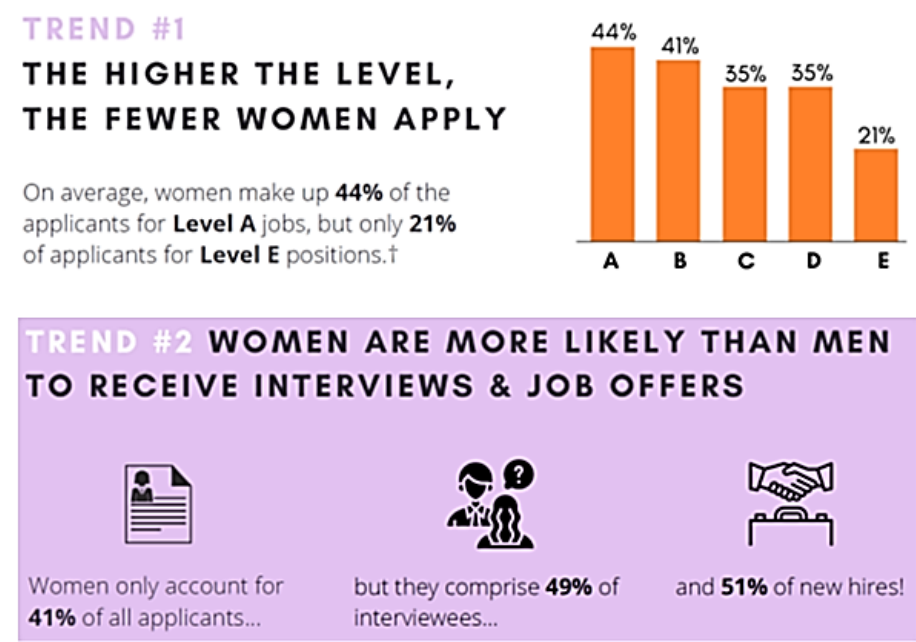

FIGURE 2: Trends on Recruitment of Women into STEMM - SAGE analysis of institutional applications

\section{DEMONSTRATING SUCCESS}

As a catalyst for change, SAGE has provided a coordinated, national approach to improving gender equity, diversity, and inclusion in STEMM across the HER sector. In the five years since its launch, it has made a bold and constructive start to addressing deeply entrenched problems. SAGE has amplified and promoted the importance of gender equity, diversity, and inclusion; it has increased transparency and strengthened institutional efforts and accountability (Putting Gender on your Agenda 2018). 


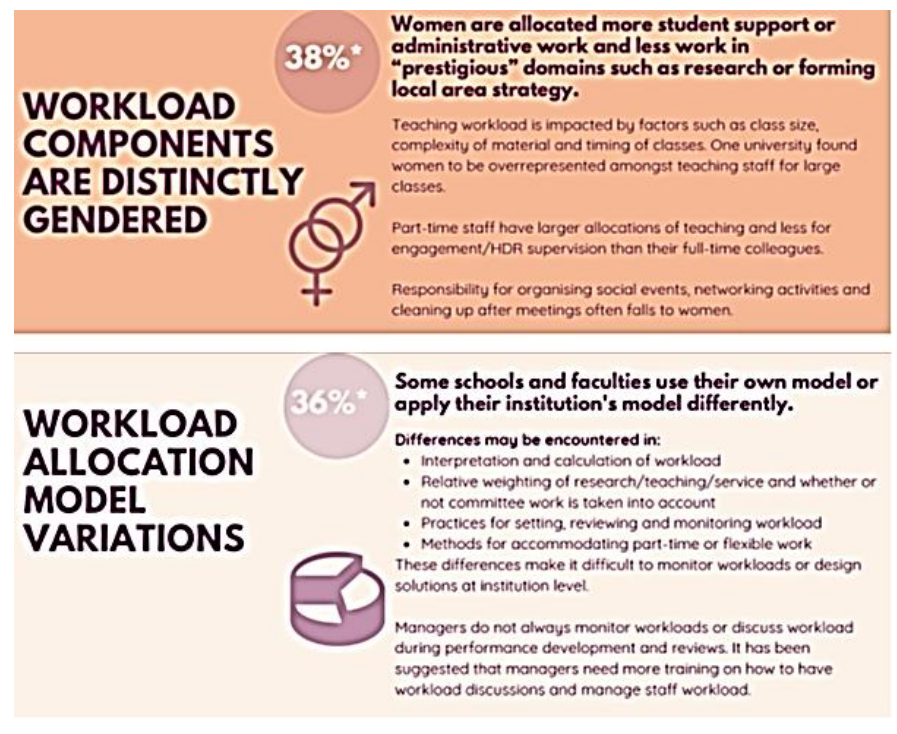

FIGURE 3: Insights on Workload Allocation - SAGE analysis of institutional applications

In this period, SAGE saw 45 institutions complete the full accreditation pathway to an Athena Swan Institutional Bronze Award (the foundational level). Of these, 43 institutions achieved the Bronze Award (ASBA 2018-2020). Despite the disruptions caused by COVID19, a major challenge for Australia's HER sector, these institutions are continuing their commitment to drive transformative change through the SAGE Athena Swan approach (Joint Sector Position Statement 2020).

Following the success of the Pilot, the Australian Government allocated further funding of $\$ 1.8$ million over the 2019-22 financial years to SAGE.

The success of the SAGE Pilot highlighted key lessons:

- Effective solutions for improving gender equity, diversity and inclusion need to be evidence-informed, systemic, and tailored to the specific institutional context. Iterative review of an organisation's structures, systems, and culture is critical, as is systematic and coordinated action underpinned by transparency and accountability.

- Leadership and cohesion, through collective effort across the sector, are critical to delivering impactful action and sustainable transformative change.

- Government backing, supported by actions of peak bodies (such as the SAGE founding academies), is a key driver of success.

- Evaluating success and measuring outcomes and impact is key to continue the drive for change - but this must be harmonised with existing national data collection and reporting systems to reduce regulatory burden.

- Enabling national, and international, collaborations to support research and knowledge creation, strengthen the evidence-base and facilitate sharing validated best practices.

Despite concerns over the administrative burden introduced by the process, and more so the risk of duplicating data collection for regulatory schemes, the consensus, supported by outcomes from the journey so far, continue to favour the SAGE Athena Swan approach. Learnings from the Pilot experience, which were formally documented in evaluations of the SAGE Pilot (Putting Gender on your Agenda 2018, and the Hartley Report 2020), led to key reforms and innovations to the approach in 2021. Most notably, the SAGE Cygnet Awards were introduced. This uniquely Australian adaptation to the Athena Swan framework was designed to drive progress and impact in key priority areas; a holistic approach that reduces administrative burden but maintains rigour.

It is in this next phase where impact will be realised and efficiently demonstrated, and where continued transparency of data and performance will drive advancement in gender equity, diversity and inclusion and sustain transformation.

\section{REFERENCES}

1. Applications for Accreditation (institutional applications).

https://www.sciencegenderequity.org.au/cohort-applications/[Reports on website] 
2. Athena Swan Charter. Advance HE. https://www.advance-he.ac.uk/. [Charter documents on website].

3. Athena Swan Principles. 2021. https://www.advance-he.ac.uk/equality-charters/athena-swancharter\#principles

4. Higher education sector commits to preserve gender equity during and after COVID-19 (2020). https://www.sciencegenderequity.org.au/? $\mathrm{s}=$ joint+sector+position+statement\&submit =Search. SAGE News, 17 June. [News article]

5. National Innovation and Science Agenda. https://www.industry.gov.au/policies-and-initiatives/boosting-innovation-and-science. [Australian Government Policy Agenda on website]

6. Putting Gender on your Agenda. 2018. Science in Australia Gender Equity. Evaluating the introduction of Athena SWAN into Australia. https://www.sciencegenderequity.org.au/wpcontent/uploads/2018/12/SAGE Report 44pp SCREEN.pdf. [Report on website]

7. SAGE Cohort Applications https://www.sciencegenderequity.org.au/cohort-applications/

8. SAGE Cygnet Awards. 2020.

https://www.sciencegenderequity.org.au/whatsage-does/\#SAGE-cygnet-awards. [Award documents on website]
9. Athena Swan Bronze Awardees (ASBA) 20182020.

https://www.sciencegenderequity.org.au/a thena-swan-bronze-awardees/

10. Australian Higher Education Joint Sector Position Statement. October 2020. Preserving Gender Equity as A Higher Education Priority During and After Covid-19.

https://www.westernsydney.edu.au/ data /assets/pdf file/0006/1799880/Australian Higher Education Joint Sector Position St atement Gender Equity COVID-19 final 16.10.20.pdf

11. Science in Australia Gender Equity Forum: Gender Equity in Science Workshop - November 2014. Summary of Workshop Findings. https://www.sciencegenderequity.org.au/wpcontent/uploads/2015/10/SAGE-Forum-Workshop-2014-Summary Report.pdf

[Report on website]

12. The Hartley Report. December 2020. https://www.sciencegenderequity.org.au/wpcontent/uploads/2020/12/The-Hartley-ReportPrecis-2020-evaluation-of-the-SAGE-peer-review-process.pdf [Report on website] 